NOTA

\title{
USO DA ADUBAÇÃO FOLIAR NITROGENADA E POTÁSSICA NO ALGODOEIRO ${ }^{(1)}$
}

\author{
MARCO ANTONIO CAMILLO DE CARVALHO ${ }^{(2,6)}$; HELDER BARBOSA \\ PAULINO $^{(2,7)}$; ENES FURLANI-JÚNIOR ${ }^{(3)}$; SALATIÉR BUZETTI ${ }^{(4)}$; MARCO \\ EUSTÁQUIO DE SÁ( ${ }^{(3)}$; MANOEL LUIZ FERREIRA DE ATHAYDE ${ }^{(5)}$
}

\section{RESUMO}

\begin{abstract}
Desenvolveu-se o experimento na região de Selvíria (MS), no ano agrícola de 1998/99, a fim de avaliar a aplicação foliar de nitrogênio e/ou potássio em complemento à adubação de semeadura na cultura do algodão (cv IAC 22), em diferentes períodos. O delineamento experimental adotado foi o de blocos ao acaso com 13 tratamentos e quatro repetições. Os tratamentos constituíram-se de uma testemunha (sem aplicação foliar) e de uma aplicação foliar de N, Ke NK, variando entre duas, quatro, seis e oito semanas após o início do florescimento. Utilizou-se a uréia como fonte de $\mathrm{N}$ e o cloreto de potássio como a de K. Em cada pulverização, aplicaram-se 250 L.ha ${ }^{-1}$ de solução, utilizando uréia na concentração de $10 \%$ e cloreto de potássio a $4 \%$. Analisaram-se as características: altura de plantas, número médio de entrenós, número médio de ramos frutíferos, número médio de capulhos, massa média de 30 capulhos, produtividade e porcentagem de fibra. Observou-se que o aumento das doses de $\mathrm{N}$ com ou sem $\mathrm{K}$, em pulverização foliar, propiciou aumento na produtividade de algodão em caroço e diminuição no rendimento de fibra. $\mathrm{O}$ uso de nitrogênio via foliar aplicado durante oito semanas após o início do florescimento proporcionou maior produtividade. Não houve resposta à aplicação de K ou NK.
\end{abstract}

Palavras-chave: nitrogênio, potássio, adubação foliar, algodoeiro.

\section{ABSTRACT \\ NITROGEN AND POTASSIUM IN FOLIAR APPLICATION ON COTTON CROP}

The present work was conducted in Selvíria county (MS-Brazil), in the agricultural year of 1998/99, for evaluating nitrogen and potassium foliar application to supplement sowing fertilization on cotton (IAC 22) crop. A randomized complete block design with 13 treatments and four replications was used. The treatments were constituted by a control (without foliar application) and nitrogen and/or potassium foliar applications two, four, six or eight weeks after beginning of flowering. The urea was used as source of $\mathrm{N}$ and potassium chloride as source of $\mathrm{K}$. Urea in the concentration of $10 \%$ and potassium chloride at $4 \%$ were applied at a rate of $250 \mathrm{~L} \cdot \mathrm{ha}^{-1}$. Height of plants, nodule number, number of reproductive branches, boll per plant, 30 boll mass, yield and fiber percentage were evaluated. It is concluded that the $\mathrm{N}$ doses increased the yield and decreased the fiber percentage. There was no evaluated response to the application of $\mathrm{K}$ or NK.

Key words: nitrogen, potassium, foliar application, cotton.

$\left({ }^{1}\right)$ Recebido para publicação em 14 de fevereiro de 2000 e aceito em 18 de outubro de 2001.

${ }^{2}$ ) Pós-graduando do Departamento de Fitotecnia e Tecnologia de Alimentos e Socioeconomia, Faculdade de Engenharia de Ilha Solteira, Universidade Estadual Paulista (FEIS/UNESP), Caixa Postal 31, 15385-000 Ilha Solteira (SP). E-mail: carvalho@agr.feis.unesp.br

$\left.{ }^{3}\right)$ Departamento de Fitotecnia, Faculdade de Ciências Agrárias e Veterinárias (FCAV), UNESP, Via de acesso Prof. Paulo Donato Castellane, 14884-000 Jaboticabal (SP).

( $\left.{ }_{5}^{4}\right)$ Departamento de Ciência do Solo e Engenharia Rural, FEIS/UNESP.

$\left({ }^{5}\right)$ Bolsista da FAPESP.

( ${ }^{6}$ Bolsista da CAPES. 
A maioria dos produtores de algodão reconhece a necessidade de um programa correto de manejo de nutrientes aplicados ao solo para alcançar as metas de produtividade; sabe-se pouco sobre as situações nas quais os fertilizantes foliares podem complementar os fertilizantes no solo, visando ao aumento da eficiência de uso do nutriente, da produtividade e do lucro. A absorção de nutrientes aplicados ao solo pode ser limitada por muitas condições: grande carga de capulhos em rápido desenvolvimento e, concomitantemente, declínio do sistema radicular ativo; redução da atividade radicular causada pela compactação do solo; acidez ou nematóides; falta temporária de umidade no solo, o que limita a difusão de nutrientes no solo; atividade radicular reduzida no enchimento dos capulhos, ou doenças. O conhecimento da interação desses fatores com a nutrição da planta pode ser útil na determinação dos benefícios potenciais da adubação foliar com $\mathrm{N}$ e $\mathrm{K}$ em programas de nutrição do algodoeiro (SNYDER, 1998).

Existem alguns trabalhos demonstrando vantagem na utilização de adubos nitrogenados via foliar em complemento à adubação tradicional nos sulcos, dentre os quais os de Buendia e NEPTUNe (1971); SABINO et al. (1993 e 1994) e SNYDER (1998). A aplicação de N no solo, geralmente, é desaconselhada depois das primeiras semanas do florescimento, pois aumenta os riscos associados ao crescimento vegetativo luxuriante, à maturação retardada e dificuldades com desfolha e preparação da colheita e ao aumento do período de exposição da planta aos insetos.

Quanto ao potássio, existem poucos trabalhos sobre a sua utilização em pulverização foliar no algodoeiro, necessitando, assim, de maiores estudos, pois no caso de deficiência de $K$, as raízes sofrem primeiro, seguidas pelos tecidos acima do solo. Desse modo, até que sejam observados os sintomas de deficiência de $\mathrm{K}$ no dossel superior da planta, a falta de $\mathrm{K}$ pode ter afetado os tecidos foliares mais velhos bem como as raízes (SNYDER, 1998).

Tendo em vista a obrigatoriedade do uso, na cultura do algodão, de um esquema definido para o controle de pragas, que inclui várias pulverizações durante o ciclo da cultura, tem-se a oportunidade de conjugar as duas práticas - adubação e controle de pragas - em uma só aplicação.

O presente trabalho teve como objetivo avaliar o efeito da aplicação foliar de nitrogênio e/ou potássio em complemento à adubação de semeadura na cultura do algodão, em diferentes períodos.

\section{Material e Métodos}

O experimento foi desenvolvido no ano agrícola de 1998/99 em área pertencente à Faculdade de Engenharia - Câmpus de Ilha Solteira - UNESP, localizada no município de Selvíria (MS). O solo do local foi classificado por Demattê (1980) como LATOSSOLO VERMELHO-ESCURO, textura argilosa.

As características químicas do solo, determinadas antes da instalação do ensaio, apresentaram os seguintes valores: 5,1 de $\mathrm{pH}$ em $\mathrm{CaCl}_{2} ; 23,0$ g.dm ${ }^{-3}$ de matéria orgânica; $17 \mathrm{mg} . \mathrm{dm}^{-3}$ de $\mathrm{P}_{\text {resina; }} ; 18,0 ; 21,0 ; 13,0$; 31,0 e $0,5 \mathrm{mmol}_{\mathrm{c}} \cdot \mathrm{dm}^{-3}$, respectivamente, de $\mathrm{K}, \mathrm{Ca}, \mathrm{Mg}$, $\mathrm{H}+\mathrm{Al}$ e $\mathrm{Al}$, e $54 \%$ de saturação por bases ( $\mathrm{V} \%)$. Realizou-se a semeadura em 8/12/1998, com 10 sementes deslintadas por metro da cultivar IAC 22, visando ao estande final de 88.000 plantas por hectare, com espaçamento de 0,90 metro.

A adubação de semeadura foi de $10 \mathrm{~kg}^{-h a^{-1}}$ de N, 100 kg.ha ${ }^{-1}$ de $\mathrm{P}_{2} \mathrm{O}_{5}$ e 50 kg.ha ${ }^{-1}$ de $\mathrm{K}_{2} \mathrm{O}$, respectivamente, na forma de sulfato de amônio, superfosfato simples e cloreto de potássio. O delineamento experimental adotado foi o de blocos ao acaso com 13 tratamentos e quatro repetições. Os tratamentos consistiram da aplicação semanal via foliar de N, Ke NK, a partir do início do florescimento. Caracterizou-se como início do florescimento a presença de uma flor aberta em $50 \%$ das plantas de cada parcela, o que ocorreu aos 44 dias após a emergência das plantas (DAE). Realizaram-se as aplicações da seguinte forma: testemunha (sem adubação foliar); $\mathrm{N}_{1}=$ aplicação de $\mathrm{N}$ aos 44 e $51 \mathrm{DAE} ; \mathrm{N}_{2}=$ aplicação de $\mathrm{N}$ aos 44, 51, 58 e $65 \mathrm{DAE} ; \mathrm{N}_{3}=$ aplicação de $\mathrm{N}$ aos 44, 51, 58, 65, 72, e $79 \mathrm{DAE} ; \mathrm{N}_{4}=$ aplicação de $\mathrm{N}$ aos 44, 51, 58, 65, 72, 79, 86 e 93 DAE; $\mathrm{K}_{1}$ = aplicação de $\mathrm{K}$ aos 44 e 51 DAE; $K_{2}=$ aplicação de $\mathrm{K}$ aos 44, 51, 58 e 65 DAE; $K_{3}=$ aplicação de $K$ aos 44, 51, 58, 65, 72 e 79 DAE; $\mathrm{K}_{4}=$ aplicação de $\mathrm{N}$ aos 44, 51, 58, 65, 72, 79, 86 e 93 DAE; $\mathrm{N}_{1} \mathrm{~K}_{1}=$ aplicação de NK aos 44 e 51 DAE; $\mathrm{N}_{2} \mathrm{~K}_{2}=$ aplicação de $\mathrm{NK}$ aos 44, 51, 58 e $65 \mathrm{DAE} ; \mathrm{N}_{3} \mathrm{~K}_{3}=$ aplicação de NK aos 44, 51, 58, 65, 72 e $79 \mathrm{DAE} ; \mathrm{N}_{4} \mathrm{~K}_{4}=$ aplicação de NK aos 44, 51, 58, 65, 72, 79, 86 e 93 DAE.

As aplicações foliares foram realizadas com pulverizador costal, tendo como fonte de $\mathrm{N}$ a uréia (10\%) e o cloreto de potássio $(4 \%)$ como fonte de $\mathrm{K}$, aplicando-se um volume de calda de 250 L.ha ${ }^{-1}$ nos finais de tarde. As pulverizações foliares ocorreram nos dias $27 / 1 ; 3 / 2$; 10/2; 17/2; 24/2; 3/3; 10/3 e 17/3/1999, sendo aplicadas as seguintes quantidades de $\mathrm{N}$ e $\mathrm{K}_{2} \mathrm{O}: \mathrm{N}_{1}=22,5$ kg.ha ${ }^{-1}$ de N; $\mathrm{N}_{2}=45,0 \mathrm{~kg} \cdot \mathrm{ha}^{-1}$ de N; $\mathrm{N}_{3}=67,5 \mathrm{~kg} \cdot \mathrm{ha}^{-1}$ de $\mathrm{N} ; \mathrm{N}_{4}=90,0 \mathrm{~kg} \cdot \mathrm{ha}^{-1}$ de N; $\mathrm{K}_{1}=12 \mathrm{~kg} \cdot \mathrm{ha}^{-1}$ de $\mathrm{K}_{2} \mathrm{O} ; \mathrm{K}_{2}=$ $24 \mathrm{~kg} \cdot \mathrm{ha}^{-1} \mathrm{de}_{2} \mathrm{O} ; . \mathrm{K}_{3}=36 \mathrm{~kg} \cdot \mathrm{ha}^{-1} \mathrm{de}_{2} \mathrm{O} ; \mathrm{K}_{4}=48 \mathrm{~kg} \cdot \mathrm{ha}^{-1}$ de $\mathrm{K}_{2} \mathrm{O} ; \mathrm{N}_{1} \mathrm{~K}_{1}=22,5 \mathrm{~kg} \cdot \mathrm{ha}^{-1}$ de $\mathrm{N}+12 \mathrm{~kg} \cdot \mathrm{ha}^{-1}$ de $\mathrm{K}_{2} \mathrm{O}$; $\mathrm{N}_{2} \mathrm{~K}_{2}=45,0 \mathrm{~kg} \cdot \mathrm{ha}^{-1}$ de $\mathrm{N}+24 \mathrm{~kg} \cdot \mathrm{ha}^{-1}$ de $\mathrm{K}_{2} \mathrm{O}$; 
$\mathrm{N}_{3} \mathrm{~K}_{3}=67,5 \mathrm{~kg} \cdot \mathrm{ha}^{-1}$ de $\mathrm{N}+36 \mathrm{~kg} \cdot \mathrm{ha}^{-1}$ de $\mathrm{K}_{2} \mathrm{O}$ e $\mathrm{N}_{4} \mathrm{~K}_{4}=90,0 \mathrm{~kg} \cdot \mathrm{ha}^{-1}$ de $\mathrm{N}+48 \mathrm{~kg} \cdot \mathrm{ha}^{-1}$ de $\mathrm{K}_{2} \mathrm{O}$.

As parcelas constituíram-se de 5 linhas de $5 \mathrm{~m}$, sendo cada uma delas distante $3 \mathrm{~m}$ da parcela subseqüente. Realizaram-se as avaliações nas três linhas centrais, desprezando-se $0,5 \mathrm{~m}$ em ambas as extremidades da parcela.

Por ocasião da colheita, em 10 plantas da área útil de cada parcela, determinaram-se: a) altura de plantas distância entre o colo e o ápice da haste principal de cada planta, medindo-se com fita graduada em milímetros; b) número médio de entrenós - contaram-se todos os entrenós da haste principal expressando-se os valores médios; c) número médio de ramos frutíferos - contaram-se todos os ramos frutíferos oriundos da haste principal; d) número médio de capulhos por planta contaram-se todos os capulhos de cada planta, expressando os valores médios; e) massa média de capulhos coletaram-se 30 capulhos do terço mediano de cada planta; f) produtividade de algodão em caroço - colheramse as plantas das duas linhas centrais de cada parcela, desprezando-se $0,5 \mathrm{~m}$ de ambas as extremidades e, g) porcentagem de fibra - após o descaroçamento dos 30 capulhos, determinou-se a porcentagem de fibra pela relação peso de fibra/peso de capulho.

Os teores totais de macronutrientes nas folhas amostradas foram determinados conforme SILVA e RAIJ (1996) em 30 plantas por parcela, utilizando-se a determinação descrita por MALAVOLTA et al. (1997).

Os resultados obtidos das características agronômicas foram submetidos à análise da variância e as médias, comparadas pelo teste de Duncan $(\mathrm{P}<5 \%)$, segundo o método de CAMPOS (1984).

\section{Resultados e Discussão}

No quadro 1 estão apresentados os valores médios de altura de plantas, número de entrenós, número de ramos frutíferos, número de capulhos, massa de um capulho, produtividade de algodão em caroço, rendimento de fibra (\%) e coeficiente de variação $(\mathrm{CV} \%)$ dessas características. Verificou-se que para a altura de plantas, número de entrenós e de ramos frutíferos não houve diferença entre qualquer tratamento em relação à testemunha. A altura de planta está próxima àquela observada por outros autores em estudos com a cultivar IAC 22 (GodinHo et al., 1999; RIBEIRO, 1999; FARIAS et al., 1999).

Quadro 1. Valores médios de altura de plantas, número de entrenós, número de ramos frutíferos, número de capulhos, massa de capulho, produtividade e rendimento de fibra de algodão, teste F e coeficiente de variação (CV), de plantas de algodão - cultivar IAC 22 - em função da aplicação foliar de N, K e NK em diferentes períodos. Selvíria (MS), 1998/99

\begin{tabular}{|c|c|c|c|c|c|c|c|}
\hline Tratamentos & $\begin{array}{l}\text { Altura de } \\
\text { plantas }\end{array}$ & $\begin{array}{l}\mathrm{N} .^{\circ} \text { de } \\
\text { entrenós }\end{array}$ & $\begin{array}{l}\mathrm{N} .^{\circ} \text { de ramos } \\
\text { frutíferos }\end{array}$ & $\begin{array}{l}\text { Número de } \\
\text { capulhos }\end{array}$ & $\begin{array}{l}\text { Massa de } \\
\text { capulho }\end{array}$ & Produtividade & $\begin{array}{l}\text { Rendimento } \\
\text { de fibra }\end{array}$ \\
\hline & $\mathrm{cm}$ & & & & $\mathrm{g}$ & kg.ha ${ }^{-1}$ & $\%$ \\
\hline Testemunha & $1,07 \mathrm{ab}$ & $12,95 \mathrm{ab}$ & $13,35 a b$ & $8,90 \mathrm{ab}$ & $5,05 \mathrm{a}$ & $2057 \mathrm{~b}$ & $36,92 \mathrm{abc}$ \\
\hline $\mathrm{N}_{1}$ & $0,97 \mathrm{~b}$ & $12,40 \mathrm{~b}$ & $12,40 \mathrm{~b}$ & $6,30 \mathrm{~b}$ & $4,92 \mathrm{a}$ & $2028 \mathrm{~b}$ & $37,42 \mathrm{ab}$ \\
\hline $\mathrm{N}_{2}$ & $1,14 \mathrm{a}$ & $13,30 \mathrm{ab}$ & $13,60 \mathrm{ab}$ & 9,80 a & $5,05 \mathrm{a}$ & $2488 \mathrm{ab}$ & $36,57 \mathrm{abc}$ \\
\hline $\mathrm{N}_{3}$ & $1,08 \mathrm{ab}$ & $14,50 \mathrm{ab}$ & $14,85 \mathrm{a}$ & $9,30 \mathrm{ab}$ & $5,30 \mathrm{a}$ & $2263 \mathrm{ab}$ & $36,42 a b c$ \\
\hline $\mathrm{N}_{4}$ & $1,19 \mathrm{a}$ & $13,75 \mathrm{ab}$ & $14,20 \mathrm{ab}$ & $10,00 \mathrm{a}$ & 5,17 a & $2750 \mathrm{a}$ & $36,10 \mathrm{bc}$ \\
\hline $\mathrm{K}_{1}$ & $1,15 \mathrm{a}$ & $13,10 \mathrm{ab}$ & $13,65 \mathrm{ab}$ & $10,45 \mathrm{a}$ & $5,27 \mathrm{a}$ & $2093 \mathrm{~b}$ & $37,17 \mathrm{abc}$ \\
\hline $\mathrm{K}_{2}$ & $1,09 \mathrm{ab}$ & $13,55 \mathrm{ab}$ & $14,00 \mathrm{ab}$ & $8,75 \mathrm{ab}$ & $4,87 \mathrm{a}$ & $2443 \mathrm{ab}$ & 37,80 a \\
\hline $\mathrm{K}_{3}$ & $1,07 \mathrm{ab}$ & $12,75 \mathrm{ab}$ & $13,15 \mathrm{ab}$ & $9,30 \mathrm{ab}$ & $4,80 \mathrm{a}$ & $2107 \mathrm{~b}$ & $37,10 \mathrm{abc}$ \\
\hline $\mathrm{K}_{4}$ & $1,12 \mathrm{ab}$ & $13,80 \mathrm{ab}$ & $13,90 \mathrm{ab}$ & $7,60 \mathrm{ab}$ & 5,07 a & $2322 \mathrm{ab}$ & $36,65 a b c$ \\
\hline $\mathrm{N}_{1} \mathrm{~K}_{1}$ & $1,19 \mathrm{a}$ & $14,90 \mathrm{a}$ & $15,10 \mathrm{a}$ & $9,70 \mathrm{a}$ & $5,32 \mathrm{a}$ & $2314 \mathrm{ab}$ & $37,00 \mathrm{abc}$ \\
\hline $\mathrm{N}_{2} \mathrm{~K}_{2}$ & $1,15 \mathrm{a}$ & $14,50 \mathrm{ab}$ & 14,65 a & $10,25 \mathrm{a}$ & $5,12 \mathrm{a}$ & $2438 \mathrm{ab}$ & $36,70 \mathrm{abc}$ \\
\hline $\mathrm{N}_{3} \mathrm{~K}_{3}$ & $1,03 \mathrm{ab}$ & $13,60 \mathrm{ab}$ & $13,80 \mathrm{ab}$ & $9,60 \mathrm{a}$ & 5,07 a & $2424 \mathrm{ab}$ & $36,10 \mathrm{bc}$ \\
\hline $\mathrm{N}_{4} \mathrm{~K}_{4}$ & $1,12 \mathrm{ab}$ & $13,90 \mathrm{ab}$ & $14,25 \mathrm{ab}$ & $10,70 \mathrm{a}$ & 5,05 a & $2338 \mathrm{ab}$ & $35,82 \mathrm{c}$ \\
\hline CV (\%) & 8,73 & 9,45 & 9,31 & 20,48 & 6,72 & 15,55 & 2,34 \\
\hline
\end{tabular}

As médias seguidas de mesma letra, na coluna, não diferem entre si pelo teste de Duncan $(\mathrm{P}<5 \%)$. 
Para o número de capulhos, verificou-se maior destaque no tratamento $\mathrm{N}_{4} \mathrm{~K}_{4}$; o valor foi significativamente superior somente ao encontrado no tratamento $\mathrm{N}_{1}$. Também, como ocorreu nas avaliações anteriores, não foi verificada diferença significativa entre qualquer tratamento em relação à testemunha.

Quanto à massa de um capulho, não se observou diferença significativa entre os tratamentos, provavelmente, por ser uma característica genética da cultivar utilizada. Esse resultado confirma as observações de SABINO et al. (1994), os quais verificaram que a aplicação isolada de uréia via foliar não afetou essa característica.

Observou-se maior produtividade no tratamento $\mathrm{N}_{4}$, diferindo significativamente dos tratamentos $\mathrm{N}_{1}$, testemunha, $\mathrm{K}_{1}$ e $\mathrm{K}_{3}$. O nível de desenvolvimento das plantas encaixa-se em uma condição de média resposta esperada à aplicação de nitrogênio, conforme observações de SILVA (1996). Esse autor enfatiza que solos ácidos ou em vias de correção, moderadamente adubados, podem levar a esse tipo de resposta à aplicação de nitrogênio na cultura do algodoeiro.

Os níveis de produtividade preconizados por FREIRE et al. (1998), 2.550 a 3.750 kg.ha- ${ }^{-1}$ para o CentroOeste, são bastante elevados e podem ser alcançados com a utilização de tecnologia adequada. Nota-se pelos resultados que o tratamento $\mathrm{N}_{4}$ proporcionou produtividade de $2.750 \mathrm{~kg} . \mathrm{ha}^{-1}$, evidenciando a resposta da cultura à aplicação do nitrogênio. Esse fato indica, pois, que a adubação foliar em complemento à adubação de semeadura pode ser uma alternativa viável para a cultura do algodão, desde que se forneça o nutriente na dose correta e parcelada adequadamente, sobretudo devido à possibilidade de aplicação conjunta com inseticidas.

Para o rendimento de fibra, o maior valor $(37,8 \%)$ foi verificado no tratamento $K_{2}$ sendo superior significativamente aos tratamentos $\mathrm{N}_{4} \mathrm{~K}_{4}, \mathrm{~N}_{3} \mathrm{~K}_{3}$ e $\mathrm{N}_{4}$. Verificou-se a diminuição no rendimento de fibra com o aumento do número de aplicações de nitrogênio. Esses resultados confirmam as observações de SABINO et al. (1993), que encontraram efeito negativo e significativo para o rendimento de fibra com o aumento da dose de nitrogênio aplicada à cultura.

A figura 1 representa os valores médios dos teores de macronutrientes avaliados nas folhas do algodoeiro após 100 dias da emergência das plântulas.

Para o nitrogênio, nota-se que os teores foliares aumentaram gradualmente, conforme aumentou-se $o$ número de pulverizações e doses do elemento, com ou sem a presença de potássio.

Nos tratamentos em que se utilizaram somente as aplicações de potássio, não houve interferência signi- ficativa nos teores foliares de nitrogênio. Os teores foliares de fósforo não mostraram efeito evidente dos tratamentos.

Observou-se o acréscimo nos teores de potássio com o aumento do número de aplicações e doses do elemento; a aplicação conjunta com nitrogênio proporcionou maiores níveis do nutriente nas folhas. Os dados variaram de 4,34 a $8,58 \mathrm{~g} \cdot \mathrm{kg}^{-1}$, valores bem abaixo dos citados por SiLVA e RAIJ (1996), 15-25 g.kg-1, e por Malavolta et al. (1997), 14-16 g.kg-1 . A época da amostragem foi o fator preponderante nessas diferenças. Tendo em vista que o potássio é um elemento bastante móvel na planta, espera-se que as amostragens tardias forneçam valores bem abaixo daqueles obtidos nas amostragens realizadas no florescimento.

Os teores foliares de Ca apresentaram tendência de redução com o aumento do número de aplicações e das doses de nitrogênio com ou sem potássio aplicados à cultura. Já o aumento isolado do número de aplicações e das doses de potássio não proporcionou redução nos teores de cálcio. Como o Ca constitui elemento de baixa mobilidade na planta, não há muita diferença nos teores do elemento em relação à época de amostragem.

Para o magnésio, nota-se que o maior número de pulverizações e maiores doses de nitrogênio proporcionaram redução nos teores nas folhas tanto para os tratamentos que receberam apenas adubação nitrogenada, como para aqueles que receberam nitrogênio e potássio conjuntamente.

A aplicação de potássio em doses superiores aos do tratamento $K_{2}$ não provocou variação nos teores foliares do elemento. Os valores observados na faixa de 4,80 a 7,73 g. $\mathrm{kg}^{-1}$ de matéria seca encontram-se na faixa adequada citada por Malavolta et al. (1997), que é de 4,00 a 5,00 g. $\mathrm{kg}^{-1}$ de matéria seca.

Os teores foliares de enxofre dos diferentes tratamentos foram superiores aos valores da testemunha, os quais se encontram na faixa de 4,06 a 5,79 g. $\mathrm{kg}^{-1}$ de matéria seca, acima dos níveis adequados, sugeridos por Malavolta et al. (1997), que são de 2,0 a 3,0 g. $\mathrm{kg}^{-1}$ de matéria seca no início do florescimento. Esse fato deve-se, provavelmente, à utilização do superfosfato simples na adubação de plantio, que pode ter proporcionado maior absorção desse elemento pela cultura, aliado à baixa mobilidade do elemento na planta, o que sempre propicia o aumento na concentração com o desenvolvimento da cultura. 

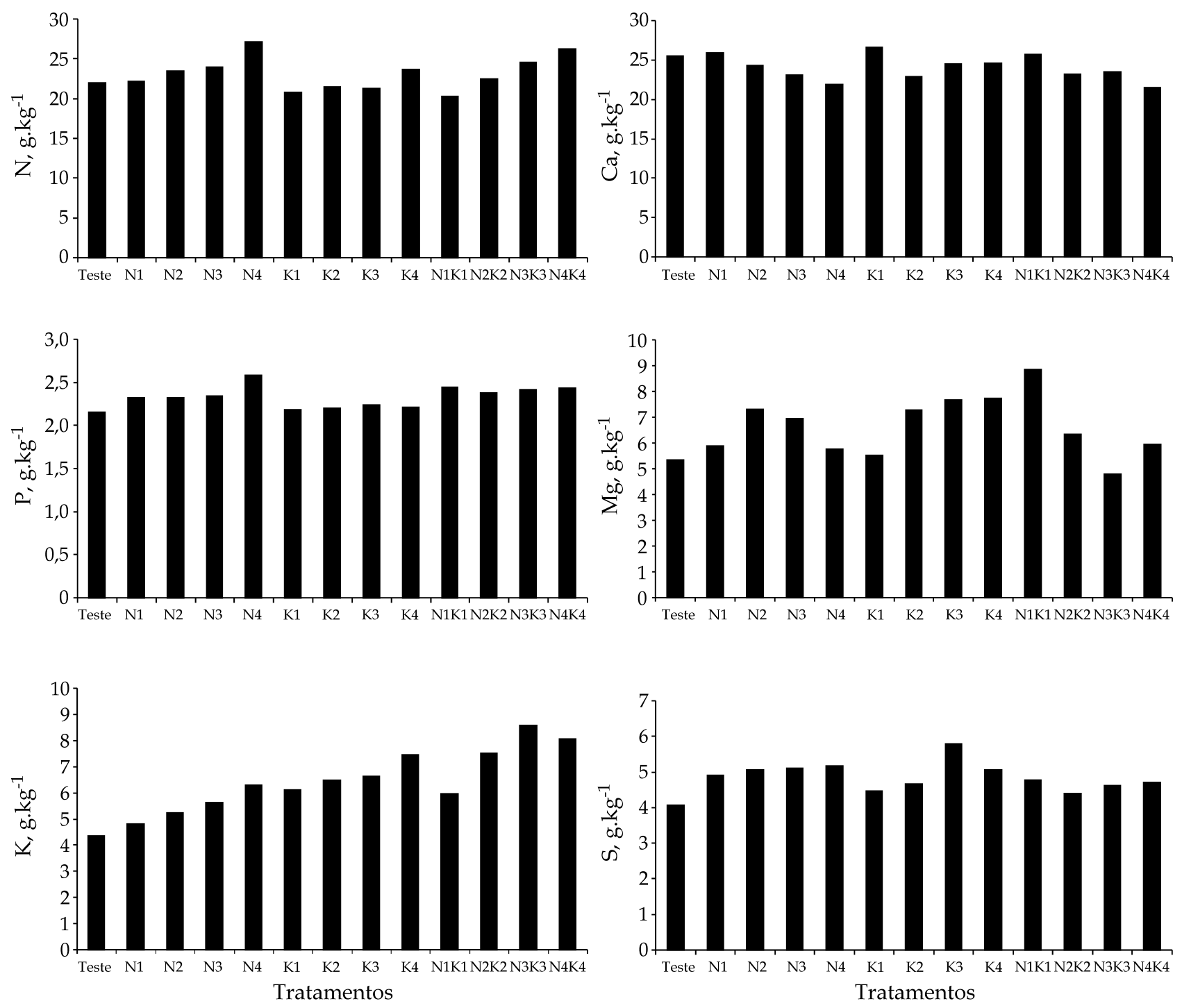

Figura 1. Teores médios de macronutrientes $\left(\mathrm{g} \cdot \mathrm{kg}^{-1}\right)$ em folhas do algodoeiro, cultivar IAC 22, aos 100 dias após emergência em função dos tratamentos.

\section{Conclusões}

1. $\mathrm{O}$ aumento das doses de $\mathrm{N}$ em pulverização foliar, com ou sem $\mathrm{K}$, propiciou o aumento na produtividade de algodão em caroço e diminuiu o rendimento de fibra.

2. As aplicações com $\mathrm{K}$ ou $\mathrm{N}+\mathrm{K}$ não afetaram a altura de plantas, o número médio de entrenós, o número médio de ramos produtivos por planta, o número médio de capulhos por planta, a massa média de capulhos e a produtividade de algodão em caroço.

\section{Referências Bibliográficas}

BUENDIA, J.P.L.; NEPTUNE, A.M.L. A adubação foliar do algodoeiro (Gossypium hirsutum L. var. IAC 12), com nitrogênio, fósforo e potássio avaliada pela produção e diagnose foliar. Anais da Escola Superior de Agricultura "Luiz de Queiroz", Piracicaba, v.28, p.5-31, 1971.

CAMPOS, H. Estatística aplicada à experimentação com canade-açúcar. São Paulo: Fundação de Estudos Agrários Luiz de Queiroz - FEALQ/USP, 1984. 292p.

DEMATTÊ, J.L.I. Levantamento detalhado dos solos do Câmpus Experimental de Ilha Solteira, Piracicaba: Escola Superior de Agricultura “Luiz de Queiroz", 1980. 131p.

FARIAS, F.J.C; FREIRE, E.C.; AGUIAR, P.H.; SOUSA, M.; MORESCO, E.R.; GRIDI-PAPP, I.L.; ARANTES, E.M.; GODINHO, V.P.C.; FERRAZ, C.T.; ALMEIDA, W.P.; SANTANA, J.C.F.; ANDRADE, F.P.; CARVALHO, L.P.; PEDROSA, M.B.; MARQUES, M.F. BRS Facual: cultivar de algodão com resistência múltipla a doenças para uso na agricultura familiar. In: CONGRESSO BRASILEIRO DE ALGODÃO, 2., 1999, Ribeirão Preto. 
Anais... Campina Grande: EMBRAPA-CNAPA, 1999. p.551-553.

FREIRE, E.C.; FARIAS, F.J.C.; FERRAZ, C.T. Cultivares. In: EMBRAPA-CPAO. Algodão: informações técnicas. EMBRAPA-CNPA: Campina Grande, 1998. p.85-102. (EMBRAPA-CPAO, Circular técnica, 7)

GODINHO, V.P.C.; PRADO, E.E.; UTUMI, M.M.; FREIRE, E.C.; FARIAS, F.J.C.; AGUIAR, P.H.; FERRO, G.O. Avaliação de genótipos de algodoeiro herbáceo no cerrado de Rondônia. In: CONGRESSO BRASILEIRO DE ALGODÃO, 2., 1999, Ribeirão Preto. Anais... Campina Grande: EMBRAPA-CNAPA, 1999. p.536-538.

MALAVOLTA, E.; VITTI, G.C.; OLIVEIRA, S.A. Avaliação do estado nutricional das plantas: princípios e aplicações. 2.ed. Piracicaba: POTAFOS, 1997. 319p.

RIBEIRO, J.L. Comportamento do algodoeiro herbáceo na mesorregião sul-maranhense. In: CONGRESSO BRASILEIRO DE ALGODÃO, 2., 1999, Ribeirão Preto. Anais... Campina Grande: EMBRAPA-CNAPA, 1999. p.539-541.
SABINO, J.C.; SILVA, N.M.; CARVALHO, L.H.; PETTINELLI-JÚNIOR, A.; SABINO, N.P.; KONDO, J.I. Aplicação de uréia em cobertura e via foliar na cultura do algodoeiro. Revista Brasileira de Ciência do Solo, Campinas, v.18, n.2, p.447-482, 1994.

SABINO, J.C.; SILVA, N.M.; CARVALHO, L.H.; SABINO, N.P.; KONDO, J.I.; NEPTUNE, A.M.L. Efeitos da aplicação de uréia em pulverização na cultura do algodoeiro. Revista Brasileira de Ciência do Solo, Campinas, v.17, n.1, p.61-66, 1993.

SILVA, N.M.; RAIJ, B. van. Fibrosas. In: RAIJ, B. van; CANTARELLA, H.; QUAGGIO, J.A.; FURLANI, A.M.C. (Eds.). Recomendações de adubação e calagem para o Estado de São Paulo. 2.ed. Campinas: Instituto Agronômico/Fundação IAC, 1996. cap.16, p.107-118.

SNYDER, C.S. Adubação foliar nitrogenada e potássica em algodão. Informações Agronômicas, Potafós, v.83, p.1-4, 1998. 